\title{
A Collaborative Approach to Minimize Cellphone Interruptions
}

\author{
Ashraf Khalil ${ }^{1}$ and Kay Connelly ${ }^{2}$ \\ ${ }^{1}$ Abu Dhabi University, College of Engineering \& Computer Science, United Arab Emirates \\ ashraf.khalileadu.ac.ae \\ ${ }^{2}$ Indiana University, School of Informatics, Bloomington, Indiana 47405, USA \\ connellyaindiana.edu
}

\begin{abstract}
In this paper, we present a collaborative approach to minimizing inappropriate cellphone interruptions. The approach uses Bluetooth technology to discover and communicate with the surrounding cell phones in order to read their notification profiles. The profile of the majority is assumed to be the most suitable setting for the current social environment. Cellphones running the collaborative service can automatically update their profile according to the majority profile or at least alert the user to do so. We have conducted a user study to examine the acceptability and the usefulness of the collaborative service and to incorporate users' feedback into the early design process.
\end{abstract}

\section{Introduction}

Mobile phones offer great accessibility and flexibility. No longer do people have to remain in a fixed location to carry on conversations over the phone. The benefits offered by cell phones, such as flexibility and accessibility, seem to inevitably come with the cost of increased interruption and interaction demands. Examples of inappropriate cell phone interruptions are when a cell phone ring disrupts a group activity, such as a class, meeting or movie. In large part, this mismatch between the user's context and the cell phone's behavior occurs because owners do not remember to frequently update their cell phone configuration according to the current context. The tremendous growth of cell phones' usage and their location-free nature have only magnified this problem.

Interruption caused by inappropriate notification such as ringing in a meeting can cause inconvenience, disruption and embarrassment for the owner. The effect of interruptions has been shown to be disruptive to task performance even when the interruption is ignored [1]. Interruption is not limited to the owner of the cell phone only but extends to the surrounding environment as well. Kern et al. have introduced and validated a model for interruptability wherein they distinguish between interruption of a user's environment (social interruptability) and interruption of a user him or herself (personal interruptability) [2].

\section{Motivation}

In this paper we describe an approach aimed at minimizing cell phone interruption: a collaborative technique that accepts the configuration of the majority of the surrounding cell phones as the appropriate configuration and adapts accordingly. The 
collaborative approach uses Bluetooth technology for discovering and communicating with the surrounding cell phones. The main idea behind this approach is the observation that most people in any given situation have their cell phones configured to the correct setting, or profile, for that situation. The collaborative approach capitalizes on the explosion of cell phones that are equipped with short-range Bluetooth capability, which enables them to communicate with other cell phones in the vicinity. For instance if a user in a meeting has forgotten to turn his cell phone ringer off, his cell phone can contact other cell phones in the same room and learn that most of them have their ringer off. Consequently, the cell phone can safely assume that it should also have its ringer off, and when the meeting is over the cell phone can return to its default state (ringer on) without the user having to take action. Cell phones that are equipped with Bluetooth connectivity can use that observation to automatically set their profile to fit the majority profile without user's explicit intervention or at least to remind the owner to manually switch to the right setting.

Bluetooth has many properties than make it ideal for collaborative configuration. In general, Bluetooth technology fits social interactions well and many applications and tools have been developed for social purposes [3]. Serendipity is a tool developed by the MIT Media lab that allows two different devices within the range of Bluetooth to communicate with each other if their personal profiles match [4].

\section{Methodology}

A user study was conducted in order to examine the acceptability and usefulness of the collaborative service and to incorporate users' feedback into the early design process. Due to the futuristic nature of the collaborative service, 10 participants were introduced to the service and were asked to 'pretend' it exists on their mobile phones. The participants, 2 of whom were males, were ages 19-30, were mostly students and all had either a full-time or part-time job. All participants had owned cell phones over a year.

The study was based on a five-day diary in which participants were asked to record relevant activities they engaged in each day, specifically those involving surrounding people, and describe those situations in which they think the collaborative service would be useful. The descriptions were to include the activity, location, number of surrounding people, their relationship with those people, and any other relevant context information. Semi-structured interviews were conducted at the end of the study in order to get their overall feedback, reactions and attitude towards the collaborative service.

\section{Findings}

\subsection{Acceptability and Usefulness}

On average participants reported 1 to 5 different situations per day, with an average of 3 , in which they could envision using the collaborative service. The diverse situations included official meetings, work, family gatherings, dinner, class, and exhibits. This indicates that participants considered the usefulness of the collaborative service in many situations both formal and informal. In the end-of-study interview, participants were also asked to rate the usefulness of the service on a scale of 1 to 5 , with 5 being 
the most useful. All participants rated the service 3 or higher with an overall average of 4. This high level of perceived usefulness is translated as a high level of usability, supported by the fact that all participants reported that they would use the service if their cell phones came equipped with it.

\subsection{User Control}

During the interview, we asked participants which if any specific features of the collaborative service they would like to have control over so they could personalize the service according to their particular needs. Participants reported a desire to control the following features:

- Turning the service on and off whenever they wish to.

- Controlling the frequency of scanning for new surrounding cellphones so that new situations can be discovered whenever possible. This parameter is a trade-off between conserving the battery power and discovering new social environments that require different cell phone configurations. Naturally, this will be different from one user to another depending on their lifestyle. For example, a student with a busy class schedule might need to set the discovery frequency higher than would an IT consultant who spends most of his day in the office with some sporadic meetings. The perceived cost of inappropriate interruption also plays an important role in deciding on the value for this parameter. Cell phone interruptions that might disturb a large group of people during an important meeting, such as in a classroom or a movie theater, are essential to avoid. Thus, a user might decide to increase the discovery frequency more than he or she would normally. Sixty percent of participants reported that they would choose 5 minutes as the default value between scans with an overall average of 30 minutes.

- Controlling the configuration transitions and notifications. Seventy percent of the participants reported that they would only use the service for a profile change going from loud (ringing) to quiet (vibration) but not the other way around. They reported that avoiding inappropriate interruption is their priority. One participant reported that she would use the service for both transitions while the rest reported that it would depend on the situation. As for the style of profile transition and whether it should be automatic or should notify the user and let her decide how to proceed, half of the participants reported that they would like to be notified of the profile change. The remaining half of the participants opted for automatic transition.

\subsection{Privacy and Trust}

Participants were asked during the interview whether they mind sharing their cellphone notification profile with the surrounding people. All but one answered negatively. The participant who reported some privacy concerns commented that "It is a bit disconcerting. It makes me think of conspiracy theory". All participants reported that they would trust the profiles of the surrounding people in the same way regardless of their social relations to them. One participant commented that "I would have the same level of trust regardless, because friends could have the wrong setting". 


\section{Conclusion}

We have introduced a novel collaborative approach that aims at improving the awareness of cellphones to the social context by minimizing inappropriate interruptions. Our approach is most useful in cases where a user forgets to change the notification profile of his or her cellphone. The diverse usage scenarios, together with the high acceptability rate reported by the users during the usability study, reflect that our proposed collaborative approach is both feasible and desirable.

It is essential to incorporate the above mentioned personalization features into the design of the collaborative service since different people are subjected to different social interactions and environments. Personalization provides them with the tools to customize the service according to their specific needs. Another important advantage of personalization is that it provides users with a sense of control over their devices and hence makes them more comfortable in using the collaborative service.

It is important to indicate that the collaborative approach is basically independent of the underlying communication technology as long as it supports the same features that are supported by Bluetooth, including short-range, automatic discovery, low power consumption and widespread adoption. However, from the current market share and the market trend over the last couple of years, it is very reasonable to assume that the majority of cell phones will be equipped with Bluetooth capability within the coming few years.

\section{References}

1. Cutrell, E., et al.: Notification, Disruption, and Memory: Effects of Messaging Interruptions on Memory and Performance. In: Proceedings of Interact (2001)

2. Kern, N., et al.: A model for human interruptability: experimental evaluation and automatic estimation from wearable sensors. In: Proceedings of IEEE International Symposium on Wearable Computers (2004)

3. Beale, R.: Supporting social interaction with the smartphone - designing pervasive systems. IEEE Pervasive Computing 4(2) (2005)

4. Eagle, N., et al.: Social Serendipity: Mobilizing Social Software. IEEE Pervasive Computing 4(2) (2005) 